\title{
DUSP6 protects murine podocytes from high glucose-induced inflammation and apoptosis
}

\author{
LIQIANG CHEN* ${ }^{*}$ YAOKUN WANG* ${ }^{*}$ HAIYAN LUAN, GUANGYU MA, HUIMING ZHANG and GUANG CHEN \\ School of Basic Medicine, Jiamusi University, Jiamusi, Heilongjiang 154002, P.R. China
}

Received February 5, 2020; Accepted June 15, 2020

DOI: $10.3892 / \mathrm{mmr} .2020 .11317$

\begin{abstract}
Diabetic nephropathy (DN) is one of the most severe complications that can occur in patients with diabetes, and without effective and timely therapeutic intervention, can gradually progress to renal failure. Previous studies have focused on investigating the pathogenesis of $\mathrm{DN}$; however, the role of dual-specificity phosphatase 6 (DUSP6) in DN is not completely understood. Therefore, the present study aimed to investigate the role of dual-specificity phosphatase 6 (DUSP6) in DN. DN model mice were established and the expression levels of DUSP6 in the kidney tissues and high glucose (HG)-induced murine podocytes (MPC5 cells) were determined using immunohistochemistry, reverse transcription-quantitative PCR and western blotting. In addition, the levels of reactive oxygen species (ROS) and inflammatory cytokines in MPC5 cells were analyzed using commercial assay kits or ELISA kits, respectively, and flow cytometric analysis was performed to analyze the rate of cell apoptosis. The present study indicated that DUSP6 expression levels were significantly decreased in DN model mice compared with control mice, and in HG-induced MPC5 cells compared with normal glucose-induced MPC5 cells. DUSP6 overexpression enhanced MPC5 cell viability and increased protein expression levels of cell markers, such as synaptopodin and nephrin, compared with the negative control group. DUSP6 overexpression also reduced the levels of ROS and inflammatory cytokines, including interleukin (IL)-1 $\beta$, IL-6 and tumor necrosis factor- $\alpha$ secreted by MPC5 cells under HG conditions. Moreover, compared with the HG group, cell apoptosis was inhibited by DUSP6 overexpression under HG conditions, which was further indicated by decreased expression levels of
\end{abstract}

Correspondence to: Miss Huiming Zhang or Professor Guang Chen, School of Basic Medicine, Jiamusi University, 258 Xuefu Road, Xiangyang, Jiamusi, Heilongjiang 154002, P.R. China

E-mail: zhmjms@163.com

E-mail: misschenguang75@163.com

${ }^{*}$ Contributed equally

Key words: diabetic nephropathy, inflammatory response, apoptosis, dual-specificity phosphatase 6 cleaved caspase-3 and Bax. Thus, these findings indicated that DUSP6 mediated the protection against HG-induced inflammatory response.

\section{Introduction}

Following improvements to living standards, the worldwide incidence of diabetes has increased annually, with $\sim 1 / 3$ of all patients with diabetes developing diabetic nephropathy (DN), a common microvascular complication of the disease (1). Previous evidence has suggested that function and structure podocyte injuries arise in the early stages of DN (2). For example, high glucose (HG) induces podocyte apoptosis, reduces the number of podocytes and damages the normal cell morphology (3), leading to glomerulosclerosis and ultimately, the development of severe proteinuria $(4,5)$. Both oxidative stress and the inflammatory response are closely associated with podocyte injury. Increased levels of reactive oxygen species (ROS) are produced in a HG environment, resulting in oxidative stress and damage to podocytes (6). Inflammatory cytokines, such as interleukin (IL)-1 $\beta$, IL-6, tumor necrosis factor- $\alpha$ (TNF- $\alpha$ ), are also secreted following HG stimulation, further exacerbating podocyte injury $(7,8)$. Podocyte injury is associated with the majority of pathological alterations that occur in glomerular diseases, serving as a critical factor for the progression of chronic kidney disease and an important target for clinical treatment (9).

Dual-specificity phosphatases (DUSPs) belong to a large family, which contains 25 phosphatases (10) that all contain a common phosphatase domain (11). DUSPs have been reported to mediate cell proliferation, migration and apoptosis (12-14). Accumulating evidence has demonstrated that DUSP6 serves a role in multiple types of cancer, such as glioblastoma, breast cancer and pancreatic cancer, where it displays either an oncogenic or tumor-suppressive function (15-18), which ultimately affects and determines the fate of a specific cancer (19). Another previous study suggested that DUSP6 inhibition enhanced T cell-modulated immunity in end-stage renal disease (20). In addition, it was also discovered that DUSP6 regulated the colon inflammatory response and protected the intestinal epithelium from oncogenic stress by promoting ERK1/2 activation (21). Similarly, DUSP6 overexpression restores $\beta$-amyloid-induced oxidative stress, endoplasmic reticulum stress and mitochondrial dysfunction via ERK1/2 activation in neural stem cells (22). However, the 
role of DUSP6 in DN and whether DUSP6 exerts a protective effect against podocyte injury are not completely understood. Therefore, the present study aimed to investigate the function of DUSP6 in HG-induced podocytes and to determine the underlying molecular mechanisms.

\section{Materials and methods}

Animals. A total of $26 \mathrm{C} 57 \mathrm{BL} / 6 \mathrm{~J}$ mice (male; weight, $20 \pm 2$ g; age, 6 weeks) were purchased from the Animal Experimental Center of Jilin University. All experiments were approved by the Biological and Medical Ethics Committee of Jamusi University (approval no. SYXK 2016-014). Mice were maintained in standardized conditions at $22 \pm 2^{\circ} \mathrm{C}$ with $55 \pm 5 \%$ relative humidity and 12 -h light/dark cycles, with free access to food and water.

Following a week of acclimatization, mice were randomly divided into two groups: i) Control $(n=8)$ and ii) diabetic $(n=18)$. The diabetic group (age, 7 weeks) were placed on a $60 \%$ high-fat diet for 4 weeks. Mice were intraperitoneally injected with $120 \mathrm{mg} / \mathrm{kg}$ streptozocin (STZ; Sigma-Aldrich; Merck KGaA) in pH 4.2 citrate buffer at 11 weeks old and mice received a second dose 5 days later, as previously reported (23). The control group were placed on a $10 \%$ low fat diet for 4 weeks, and received an intraperitoneal injection of citrate buffer without streptozocin at 11 weeks old and a second injection 5 days later. Animal behaviors (activity, appetite and mental status) were monitored every 2 days. At $72 \mathrm{~h}$ after the second injection, $0.1 \mathrm{ml}$ blood was collected through the tail vein. Subsequently, non-fasting blood glucose levels were assessed using an Accu-Check Compact $^{\circledR}$ glucometer (Roche Diagnostics). Mice with non-fasting blood glucose levels $\geq 16.7 \mathrm{mmol} / 1$ (300 mg/dl) were considered as diabetic model mice. The duration of the experiment was 6 weeks, including a week of adaptive feeding. Mice were placed in individual metabolic cages for 24-h urine collection prior to sacrifice. Mice were sacrificed by cervical dislocation at the end of the experiment, and death was verified by monitoring cessation of the heartbeat and responses to external stimuli. Subsequently, the kidneys were extracted.

Determination of urinary microalbumin/creatinine ratio $(U A C R)$. The urine microalbumin ELISA kit (cat. no. ml063626) and creatinine ELISA kit (cat. no. ml037582) were purchased from Shanghai Enzyme-linked Biotechnology Co., Ltd.; both urine microalbumin and creatinine levels were determined according to the manufacturers' protocols. The UACR was calculated to evaluate renal function.

Hematoxylin and eosin staining. Kidney tissue was fixed with $4 \%$ paraformaldehyde for $36 \mathrm{~h}$ at $4^{\circ} \mathrm{C}$, embedded in paraffin and cut into $4-\mu \mathrm{m}$ thick paraffin sections. The sections were subsequently deparaffinized twice using xylene for $10 \mathrm{~min}$ each at room temperature and rehydrated using a descending ethanol series. Subsequently, sections were stained with hematoxylin (nuclei) for $5 \mathrm{~min}$ at room temperature and eosin (cytoplasm) for $3 \mathrm{~min}$ at room temperature. The sections were dehydrated using an ascending alcohol series, sealed with neutral balsam (Beijing Solarbio Science \& Technology Co.,
Ltd.; cat. no. G8590) and observed using a light microscope (Olympus Corporation; magnification, x200).

Masson staining. Kidney tissue samples were fixed with $4 \%$ paraformaldehyde for $36 \mathrm{~h}$ at $4^{\circ} \mathrm{C}$, paraffin-embedded, cut into $4-\mu \mathrm{m}$ thick sections, deparaffinized twice using xylene for $10 \mathrm{~min}$ each at room temperature and rehydrated using a descending series of ethanol. Subsequently, nuclear staining was performed using Regaud hematoxylin dye for $10 \mathrm{~min}$ at room temperature. Following washing with distilled water, the sections were stained with Ponceau S staining solution for $2 \mathrm{~min}$ at room temperature. The sections were soaked with $0.2 \%$ glacial acetic acid aqueous solution for $1 \mathrm{~min}, 1 \%$ phosphomolybdate for $5 \mathrm{~min}$ and $0.2 \%$ glacial acetic acid for $2 \mathrm{~min}$ (all at room temperature). Following washing with distilled water, methyl green staining was conducted for $3 \mathrm{~min}$ at room temperature. The sections were then treated with $95 \%$ ethanol for $10 \mathrm{sec}$ to dehydrate, then $100 \%$ ethanol was used in triplicate to dehydrate the sections for $10 \mathrm{sec}$ each time. After sealing with neutral balsam, sections were observed using a light microscope (Olympus Corporation; magnification, x200).

Immunohistochemistry. Kidney tissue was fixed in $4 \%$ paraformaldehyde for $36 \mathrm{~h}$ at $4^{\circ} \mathrm{C}$, paraffin-embedded, cut into 4- $\mu \mathrm{m}$ thick sections and deparaffinized twice using xylene for $10 \mathrm{~min}$ at room temperature. The sections were then rehydrated with a descending series of ethanol. Antigen retrieval was performed by heating the sections in boiling $0.01 \mathrm{M}$ sodium citrate buffer solution ( $\mathrm{pH}$ 6.0) for $10 \mathrm{~min}$. After washing with PBS, slides were blocked with $10 \%$ goat serum (Gibco; Thermo Fisher Scientific, Inc.) for $1 \mathrm{~h}$ at room temperature. Subsequently, slides were incubated overnight at $4^{\circ} \mathrm{C}$ with an anti-DUSP6 (Abcam; cat. no. ab76310; 1:50) primary antibody. Following washing with PBS, slides were incubated with a goat anti-rabbit IgG horseradish peroxidase (HRP)-conjugated secondary antibody (BIOSS; cat. no. bs-0295G-HRP; 1:3,000) for $1 \mathrm{~h}$ at $37^{\circ} \mathrm{C}$. Stained sections were observed under a light microscope (Olympus Corporation; magnification, x200).

Cell culture and transfection. Conditionally immortalized MPC5 cells (Beijing Beina Chuanglian Biotechnology Research Institute; cat. no. BNCC337685) were cultured in RPMI-1640 medium (Hyclone; Cytiva) supplemented with $10 \%$ FBS (Gibco; Thermo Fisher Scientific, Inc.) and $50 \mathrm{IU} / \mathrm{ml}$ IFN- $\gamma$ (PeproTech, Inc.) at $33^{\circ} \mathrm{C}$ in collagen I-coated dishes (BD Biosciences). To induce differentiation, MPC5 cells were cultured for 14 days at $37^{\circ} \mathrm{C}$ without IFN- $\gamma$ and used for subsequent experiments. To induce cell injury, $2 \times 10^{5} \mathrm{MPC} 5$ cells/well were seeded into a six-well plate and incubated with different concentrations of D-glucose $(5,10,15,20,25$ or $30 \mathrm{mM}$; Sigma-Aldrich; Merck KGaA) for $24 \mathrm{~h}$ at $37^{\circ} \mathrm{C}$. For the control group, 30 mM D-mannitol (MA; Sigma-Aldrich; Merck $\mathrm{KGaA}$ ) was added into the culture medium at $37^{\circ} \mathrm{C}$ for $24 \mathrm{~h}$ to adjust the osmotic pressure to be consistent with the $30 \mathrm{mM}$ D-glucose group (24). DUSP6 overexpression (OE) plasmids (OE-DUSP6) and empty vector (negative control; NC) plasmids (OE-NC) were purchased from Shanghai Qincheng Biological Technology Co., Ltd. MPC5 cells were seeded into a 6-well plate. At 60-70\% confluence, MPC5 cells were transfected with $4 \mu \mathrm{g}$ OE-DUSP6 or OE-NC using Lipofectamine ${ }^{\circledR} 2000$ 
(Invitrogen; Thermo Fisher Scientific, Inc.). After incubation for $6 \mathrm{~h}$ at $37^{\circ} \mathrm{C}$, the medium was replaced with fresh complete medium and incubated for $48 \mathrm{~h}$ at $37^{\circ} \mathrm{C}$.

$R T$ - $q P C R$. Total RNA was extracted from MPC5 cells or kidney tissues using TRIzol ${ }^{\circledR}$ (Invitrogen; Thermo Fisher Scientific, Inc.). Total RNA was reverse transcribed into cDNA using a PrimeScript ${ }^{\mathrm{TM}}$ RT Reagent kit (Takara Bio, Inc.). The following RT temperature protocol was used: $37^{\circ} \mathrm{C}$ for $5 \mathrm{~min}$ and $85^{\circ} \mathrm{C}$ for $5 \mathrm{sec}$, then maintained at $4^{\circ} \mathrm{C}$. Subsequently, qPCR was performed using a SYBR Green PCR Master mix (Thermo Fisher Scientific, Inc.) on an ABI 7500 Real-Time PCR system (Applied Biosystems; Thermo Fisher Scientific, Inc.). The following thermocycling conditions were used for the qPCR: Initial denaturation at $95^{\circ} \mathrm{C}$ for $5 \mathrm{~min}$; followed by 40 cycles of $95^{\circ} \mathrm{C}$ for $10 \mathrm{sec}$ and $60^{\circ} \mathrm{C}$ for $30 \mathrm{sec}$; followed by $95^{\circ} \mathrm{C}$ for $15 \mathrm{sec}, 60^{\circ} \mathrm{C}$ for $60 \mathrm{sec}$ and $95^{\circ} \mathrm{C}$ for $15 \mathrm{sec}$. The following primers were used for the qPCR: DUSP6 forward, 5'-CGACTGGAATGAGAACACTGGTGG-3' and reverse, 5'-TCTAGATTGGTCTCGCAGTGCAGG-3'; and GAPDH forward, 5'-GGCCCCTCTGGAAAGCTGTG-3' and reverse, 5'-CCGCCTGCTTCACCACCTTCT-3'. The relative expression levels of each gene were quantified using the $2^{-\Delta \Delta \mathrm{Cq}}$ method (25) and normalized to the endogenous reference gene GAPDH.

Western blotting. Total protein was extracted from MPC5 cells using RIPA lysis buffer (Beyotime Institute of Biotechnology) containing phenylmethanesulfonyl fluoride and protease inhibitor. Following quantification using a bicinchoninic acid assay kit (Beyotime Institute of Biotechnology), $30 \mu \mathrm{g}$ protein/lane was separated via 10\% SDS-PAGE and transferred to PVDF membranes (EMD Millipore). Following blocking with $5 \%$ non-fat milk for $2 \mathrm{~h}$ at room temperature, the membranes were incubated overnight at $4^{\circ} \mathrm{C}$ with primary antibodies against: DUSP6 (Abcam; cat. no. ab76310; 1:1,000), nephrin (Abcam; cat. no. ab216341; 1:1,000), Bcl-2 (Abcam; cat. no. ab182858; 1:1,000), Bax (Abcam; cat. no. ab182733; 1:1,000), cleaved caspase-3 (Abcam; cat. no. ab2302; 1:1,000), caspase-3 (Abcam; cat. no. ab44976; 1:1,000), synaptopodin (Santa Cruz Biotechnology, Inc.; cat. no. sc-515842; 1: 500), phosphorylated (p)-ERK1/2 (Cell Signaling Technology, Inc.; cat. no. 9101; 1:1,000), ERK1/2 (Cell Signaling Technology, Inc.; cat. no. 4695; 1:1,000) and GAPDH (Cell Signaling Technology, Inc.; cat. no. 2118; 1:1,000). Following the primary incubation, the membranes were incubated with a goat anti-rabbit IgG HRP-conjugated $(1: 10,000)$ or goat anti-mouse IgG HRP-conjugated (BIOSS; cat. no. bs-0296G-HRP; $1: 10,000)$ secondary antibody for $1 \mathrm{~h}$ at room temperature. Protein bands were visualized using an ECL Substrate kit (BioVision, Inc.) and analyzed using Image Lab software (v2.1; Bio-Rad Laboratories, Inc.). GAPDH was used as the loading control.

Determination of $I L-1 \beta, I L-6$ and TNF- $\alpha$ levels. The cell medium of MPC5 cells was centrifuged at 2,000 x g for $5 \mathrm{~min}$ at room temperature, and the levels of IL-1 $\beta$ (cat. no. SBJ-M0027), IL-6 (cat. no. SBJ-M0044) and TNF- $\alpha$ (cat. no. SBJ-M0030) were measured using ELISA kits (Nanjing Senbega Biological Technology Co., Ltd.).
MP5 cells were lysed using RIPA lysis buffer and centrifuged at $10,000 \mathrm{x} \mathrm{g}$ for $10 \mathrm{~min}$ at $4^{\circ} \mathrm{C}$. The cell supernatant was subsequently collected to detect malondialdehyde (MDA) levels using a commercial colorimetric kit (cat. no. S0131S; Beyotime Institute of Biotechnology).

Cell Counting Kit-8 (CCK-8). MPC5 cells were seeded $\left(5 \times 10^{3}\right.$ cells/well $)$ in a 96 -well plate and cultured with $30 \mathrm{mM}$ D-glucose for $24 \mathrm{~h}$ at $37^{\circ} \mathrm{C}$. Subsequently, $10 \mu \mathrm{l} \mathrm{CCK}-8$ solution (Beyotime Institute of Biotechnology) was added to each well and incubated for $4 \mathrm{~h}$ at $37^{\circ} \mathrm{C}$, according to the manufacturer's protocol. The absorbance of each well was measured at a wavelength of $450 \mathrm{~nm}$ using a microplate spectrophotometer.

ROS staining. Fluorescent Probe-dihydroethidium (DHE; Vigorous Biotechnology (Beijing) Co., Ltd.) was used to determine cellular ROS levels. Briefly, $2 \times 10^{4}$ MPC5 cells/well were seeded into a 24-well plate and treated with DHE $(10 \mu \mathrm{M})$ for $20 \mathrm{~min}$ at $37^{\circ} \mathrm{C}$, then observed using a fluorescence microscope (Olympus Corporation; magnification, x100). The fluorescence intensity was analyzed using ImageJ software (v1.8.0; National Institutes of Health).

Flow cytometry assay. MPC5 cells were washed with PBS and harvested using $0.25 \%$ trypsin (Beyotime Institute of Biotechnology). Subsequently, cells were centrifuged (1000 x g; 5 min; room temperature), collected and re-suspended in PBS. Cells $\left(5 \times 10^{4}-1 \times 10^{5}\right)$ were centrifuged $(200 \times \mathrm{g} ; 5 \mathrm{~min}$; room temperature) and re-suspended in $300 \mu 1$ Annexin-V binding buffer (Biomars). Subsequently, cells were stained with $5 \mu 1$ Annexin-V-FITC (Biomars) for $15 \mathrm{~min}$ at room temperature and $5 \mu \mathrm{l}$ propidium iodide (Biomars) for $10 \mathrm{~min}$ on ice in the dark. Apoptotic cells were detected by flow cytometry (BD FACSCalibur; BD Biosciences) and analyzed using Accuri C6 software (v1.0.264.21; BD Biosciences). FITC-positive (Q2 and Q3) cells were considered as apoptotic cells (early and late apoptosis).

Statistical analysis. Data are presented as the mean \pm SD of at least three replicates. Comparisons between two groups were analyzed using an unpaired Student's t-test. Comparisons among multiple groups were analyzed using one-way ANOVA followed by Tukey's post hoc test. Statistical analyses were performed using SPSS software (v19.0; IBM Corp.). $\mathrm{P}<0.05$ was considered to indicate a statistically significant difference.

\section{Results}

DUSP6 expression levels are reduced in diabetes-induced DN model mice. At $72 \mathrm{~h}$ after the final STZ injection, non-fasting blood glucose levels were assessed. A total of 16 mice in the diabetic group displayed a blood glucose level $\geq 16.7 \mathrm{mmol} / \mathrm{l}$ and were therefore considered diabetic model mice. The blood glucose levels of the remaining two mice in the diabetic group did not exceed $16.7 \mathrm{mmol} / \mathrm{l}$; therefore, these mice were excluded from the present study. In the diabetic group, a mean blood glucose level of $20 \mathrm{mmol} / 1$ was reported (Fig. 1A), and the mice displayed a significantly increased UACR compared with the control group (Fig. 1B). The morphological examination of the kidneys of the control group indicated a normal 
A

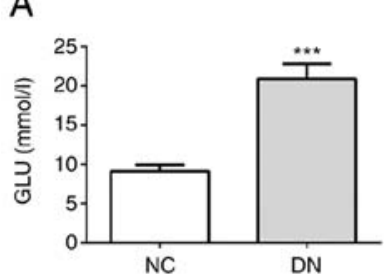

D

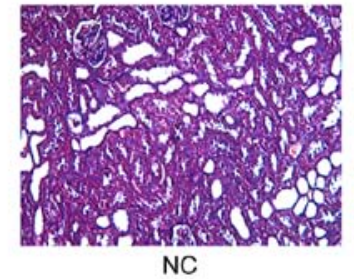

F

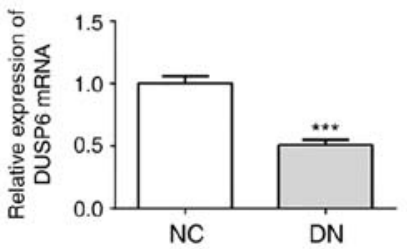

B
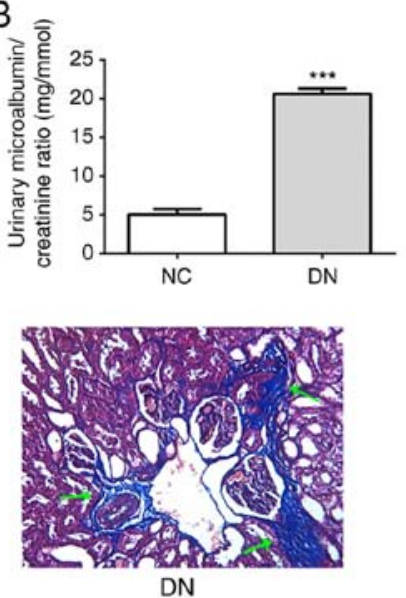

G

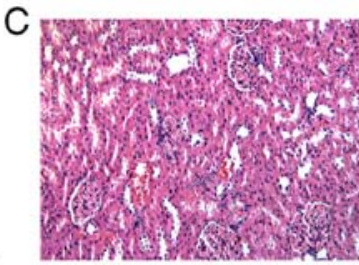

NC

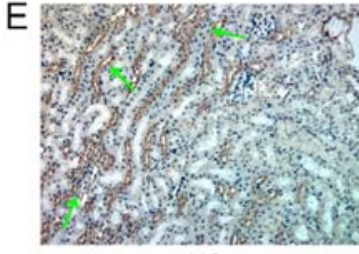

NC

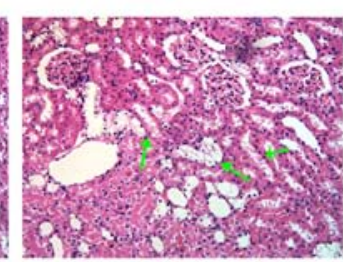

DN

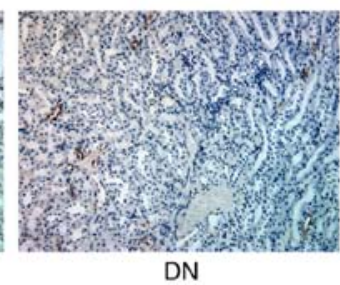

DN

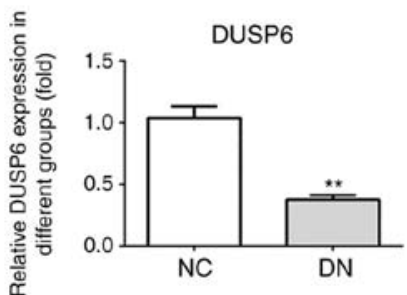

Figure 1. DUSP6 expression levels are reduced in diabetes-induced DN model mice. (A) Blood glucose levels. (B) Urinary microalbumin/creatinine ratio. (C) Representative images of hematoxylin and eosin staining of paraffin-embedded kidney tissues (magnification, x200). Green arrows indicate pathological changes. (D) Representative images of Masson staining of paraffin-embedded kidney tissues (magnification, x200). Green arrows indicate areas of fibrosis. (E) DUSP6 protein expression in kidney tissues was detected by immunohistochemistry (magnification, x200). Green arrows indicate DUSP6-positive areas DUSP6 (F) mRNA and (G) protein expression levels in kidney tissues. ${ }^{* *} \mathrm{P}<0.01$ and ${ }^{* * *} \mathrm{P}<0.001$ vs. NC. DN, diabetic nephropathy; DUSP6, dual-specificity phosphatase 6; NC, negative control; GLU, glucose.

histology, whereas six mice in the diabetic group $(n=16)$ displayed an extended glomerular area and mesangial matrix expansion (Fig. 1C). Masson staining also indicated collagen fiber accumulation in the diabetic group, suggesting the occurrence of renal fibrosis (Fig. 1D). The pathological alterations to the kidneys of the six mice in the diabetic group indicated the successful establishment of the DN mouse model, as previously reported (26). Subsequently, the expression levels of DUSP6 in diabetic model mice with significant pathological alterations in the kidneys were analyzed. The results indicated that increased DUSP6 expression levels were observed in the control mice compared with the DN model mice, which was consistent with the RT-qPCR and western blotting results (Fig. 1F and G). Overall, the results suggested that DUSP6 expression levels may be decreased in DN model mice.

DUSP6 expression levels are decreased in HG-induced murine podocytes. It was previously demonstrated that DUSP6 expression levels were downregulated in the kidneys of DN model mice, which suggested that podocyte functions may be closely linked to DN (2). D-glucose is used to simulate a HG environment in vivo $(24,27)$; therefore, the present study used a gradient of D-glucose concentrations (5-30 mM) to treat MCP5 murine podocytes. The results indicated that $30 \mathrm{mM}$ D-glucose was the dose that most effectively inhibited DUSP6 mRNA and protein expression levels compared with the $5 \mathrm{mM}$ D-glucose group (Fig. 2A and B). Subsequently, MPC5 cells were induced with $30 \mathrm{mM}$ D-glucose (HG) for different incubation periods. The results indicated that the optimum incubation period for D-glucose-mediated stimulation of MPC5 podocytes was $24 \mathrm{~h}$, as DUSP6 expression levels were reduced to the lowest levels at $24 \mathrm{~h}$ compared with the $0 \mathrm{~h}$ group (Fig. 2C). Moreover, compared with the normal glucose (NG) group (5 mM D-glucose), MA did not significantly alter the expression levels of DUSP6. By contrast, HG significantly decreased DUSP6 mRNA and protein expression levels compared with the NG group (Fig. 2D and E). Altogether, the results suggested that DUSP6 expression levels may be reduced in HG-induced MPC5 cells.

DUSP6 overexpression protects against HG-induced podocyte injury. To further elucidate the role of DUSP6 in podocyte injury, OE-DUSP6 and OE-NC were constructed and transfected into MPC5 cells. RT-qPCR and western blotting were performed to assess transfection efficiency. DUSP6 mRNA and protein expression levels were significantly increased in the OE-DUSP6 group compared with the OE-NC group (Fig. 3A and B). Subsequently, MPC5 cell viability and the expression of specific markers (synaptopodin and nephrin) were also investigated. MPC5 cell viability was significantly decreased by HG compared with the NG group; however, OE-DUSP6 reversed HG-mediated effects on cell viability (Fig. 3C). Similarly, synaptopodin and nephrin protein expression levels were significantly reduced in HG-treated MPC5 cells compared with NG-treated cells, whereas DUSP6 overexpression reversed HG-mediated downregulation of protein expression (Fig. 3D). In summary, the results indicated that DUSP6 alleviated HG-induced cell injury. 
A

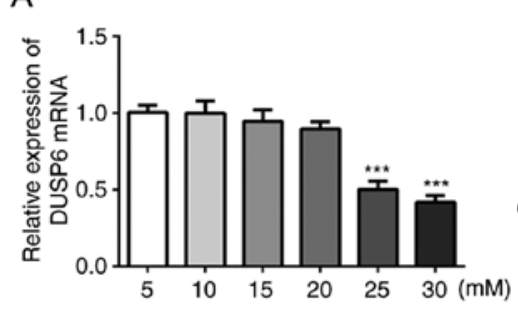

C
B
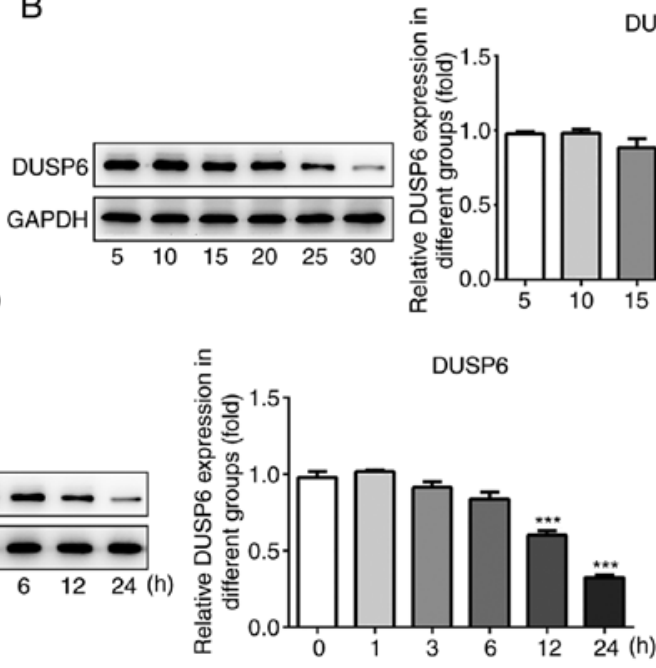

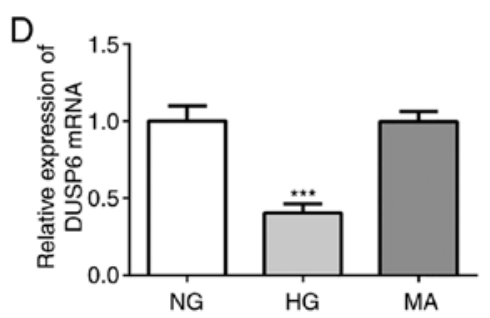

E
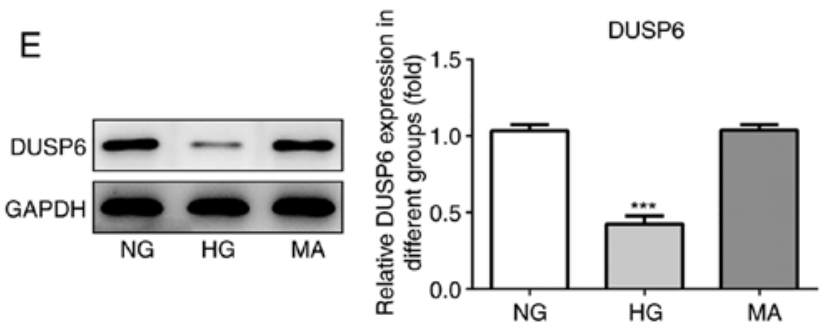

Figure 2. DUSP6 expression levels are decreased in HG-induced murine podocytes. DUSP6 (A) mRNA and (B) protein expression levels in MPC5 cells stimulated with different concentrations of D-glucose. ${ }^{* * * *} \mathrm{P}<0.001 \mathrm{vs.} 5 \mathrm{mM}$ D-glucose. (C) DUSP6 protein expression levels in MPC5 cells stimulated with $30 \mathrm{mM}$ D-glucose for different incubation periods. ${ }^{* * *} \mathrm{P}<0.001$ vs. $0 \mathrm{~h}$. DUSP6 (D) mRNA and (E) protein expression levels in MPC5 cells treated with 5 mM D-glucose, $30 \mathrm{mM}$ D-glucose or MA for $24 \mathrm{~h} .{ }^{* * *} \mathrm{P}<0.001$ vs. NG. DUSP6, dual-specificity phosphatase 6; HG, high glucose; NG, normal glucose; MA, D-mannitol.

A

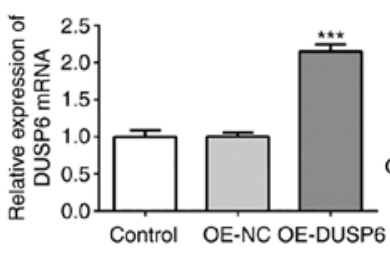

D

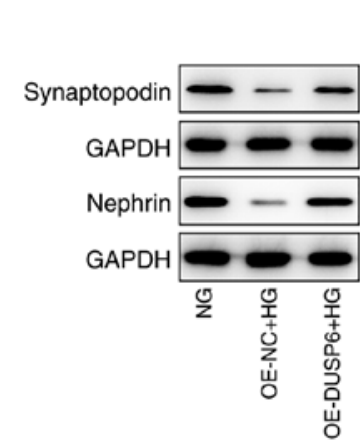

B
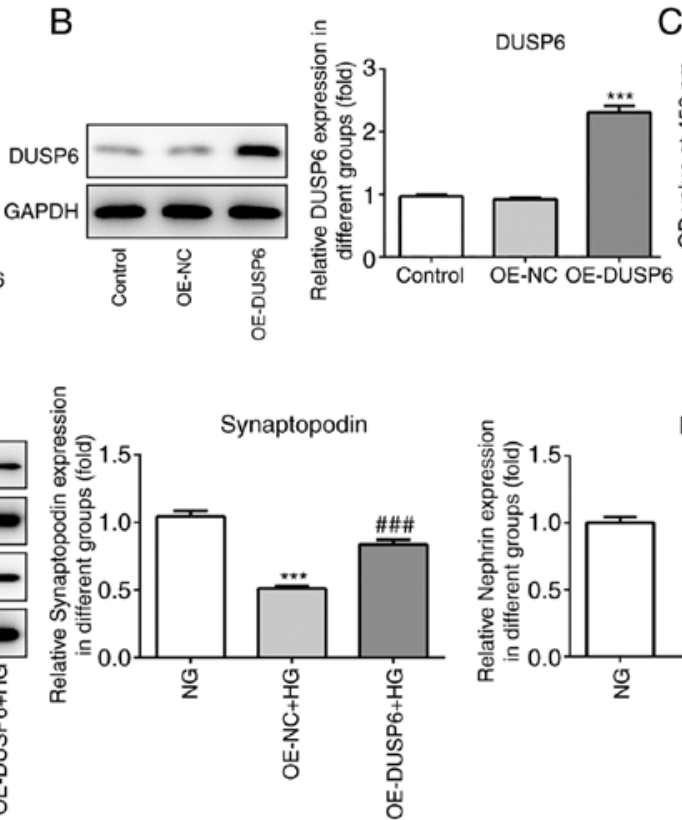

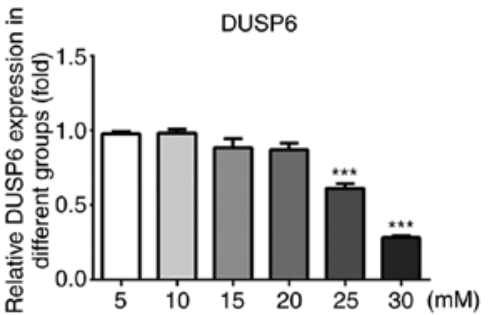




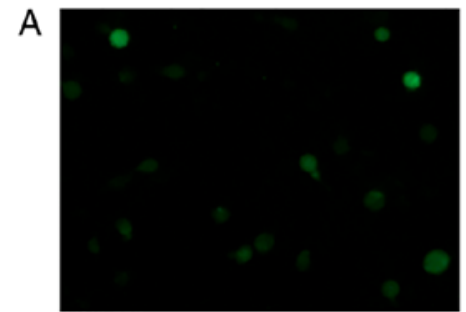

NG

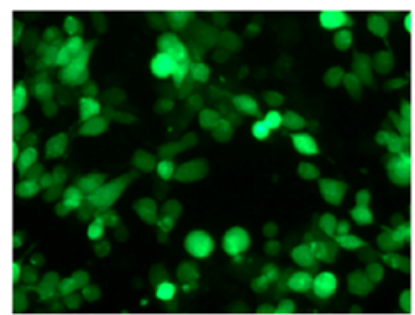

$\mathrm{OE}-\mathrm{NC}+\mathrm{HG}$

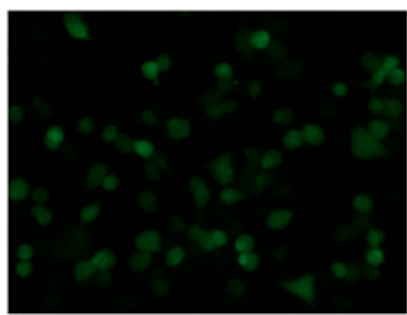

OE-DUSP6+HG
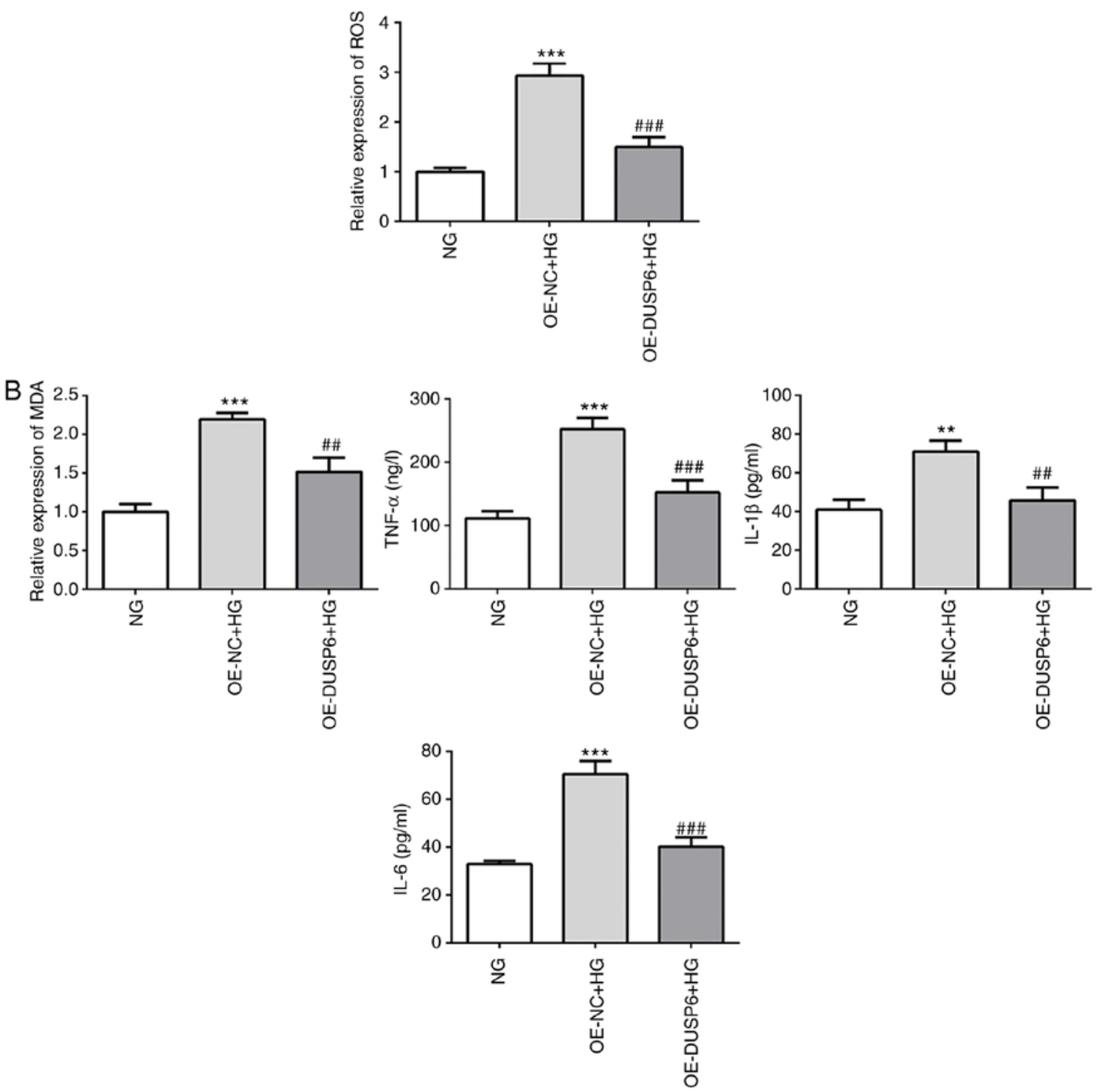

Figure 4. DUSP6 overexpression attenuates HG-induced oxidative stress and inflammatory responses. (A) ROS levels were determined by ROS staining using a DHE-probe and the relative fluorescence intensity was semi-quantified (magnification, x100). (B) The levels of MDA, IL-1 $\beta$, IL- 6 and TNF- $\alpha$ were measured using ELISA kits. ${ }^{* *} \mathrm{P}<0.01$ and ${ }^{* * *} \mathrm{P}<0.001$ vs. NG; ${ }^{\# \#} \mathrm{P}<0.01$ and ${ }^{\# \# \#} \mathrm{P}<0.001$ vs. OE-NC + HG. DUSP6, dual-specificity phosphatase 6; HG, high glucose; ROS, reactive oxygen species; MDA, malondialdehyde; IL, interleukin; TNF, tumor necrosis factor; NG, normal glucose; OE, overexpression; NC, negative control; DHE, dihydroethidium.

were reversed by DUSP6 overexpression (Fig. 4A). MDA, a cellular oxidative stress product, was also significantly reduced in DUSP6-overexpression MPC5 cells under HG conditions compared with the $\mathrm{OE}-\mathrm{NC}+\mathrm{HG}$ group (Fig. 4B). In addition, the levels of TNF- $\alpha$, IL- $1 \beta$ and IL- 6 were determined using ELISA kits. DUSP6 overexpression reversed HG-induced upregulation of inflammatory cytokine levels (Fig. 4B). The results indicated that DUSP6 overexpression may relieve oxidative stress and the inflammatory response in MPC5 cells.
DUSP6 overexpression alleviates HG-induced podocyte apoptosis. Subsequently, the effect of DUSP6 on podocyte apoptosis was investigated. In a previous study, the HG environment promoted podocyte apoptosis, leading to glomerulosclerosis and severe proteinuria $(4,5)$. The effective prevention of podocyte apoptosis is crucial for the treatment of DN. HG-induced MPC5 cells displayed an increased rate of apoptosis compared with the NG group; however, DUSP6 overexpression significantly reduced HG-induced apoptosis 

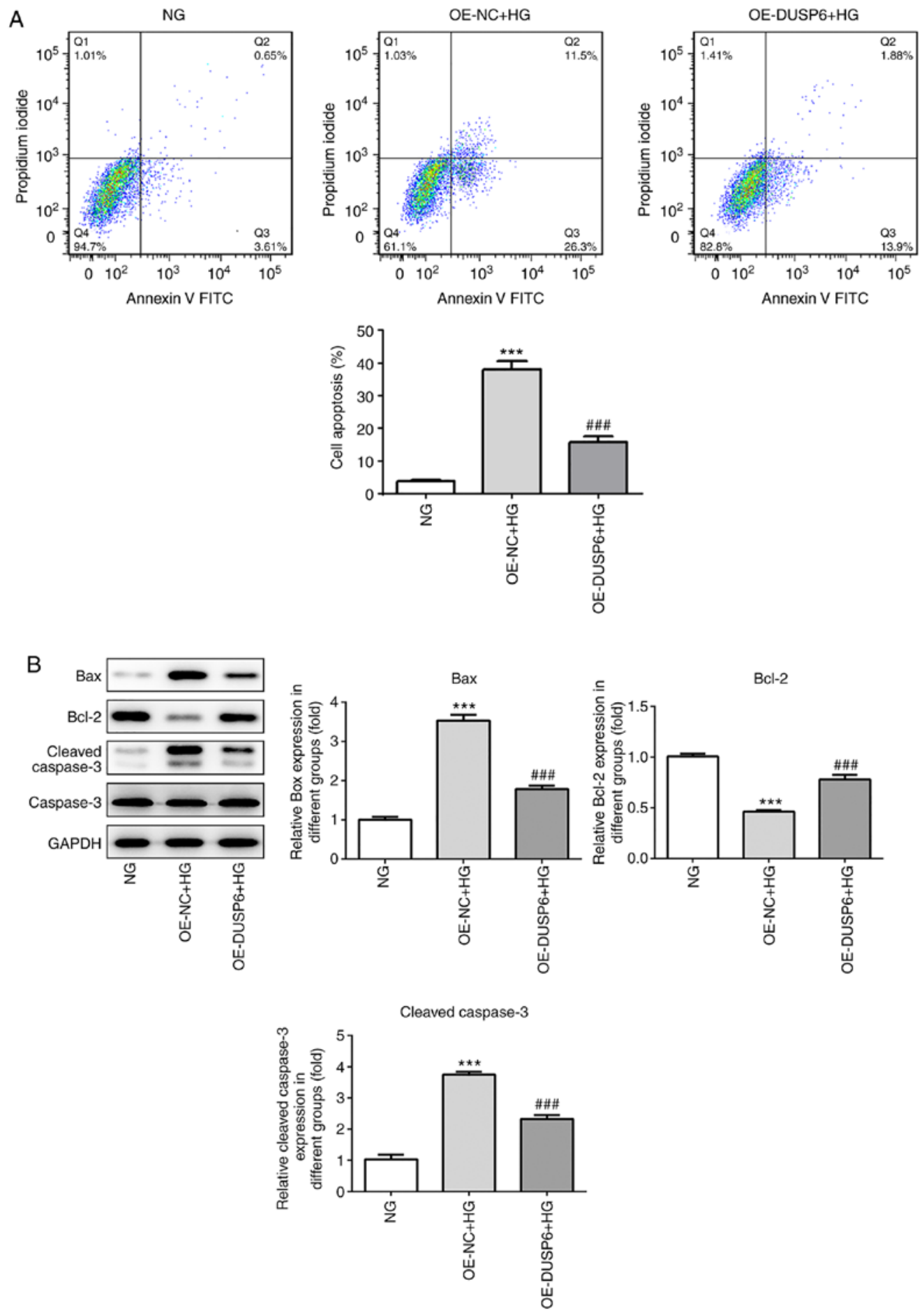

Figure 5. DUSP6 overexpression alleviates HG-induced podocyte apoptosis. (A) Apoptotic cells were detected via flow cytometry. (B) The protein expression levels of Bcl-2, Bax, cleaved caspase-3 and caspase-3. ${ }^{* * *} \mathrm{P}<0.001$ vs. NG; ${ }^{\# \# *} \mathrm{P}<0.001$ vs. OE-NC + HG. DUSP6, dual-specificity phosphatase 6; HG, high glucose; NG, normal glucose; $\mathrm{OE}$, overexpression; $\mathrm{NC}$, negative control.

(Fig. 5A). Consistently, HG significantly upregulated Bax protein expression levels compared with the NG group, but DUSP6 overexpression reversed HG-mediated alterations to Bax expression. By contrast, Bcl-2 expression levels displayed the opposite trend. Cleaved caspase-3 expression levels were significantly upregulated in the HG group compared with the NG group, which was reversed by DUSP6 overexpression (Fig. 5B). Collectively, the results suggested that DUSP6 may prevent HG-induced podocyte apoptosis.
ERK1/2 activation is associated with the role of DUSP6 in murine podocytes. Finally, the potential mechanism underlying the protective effects of DUSP6 in MPC5 cells was investigated. DUSPs tightly mediate the spatiotemporal activity and inactivation of mitogen-activated protein kinases (MAPKs) (28). As members of the MAPK family, ERK1/2 are highly homologous and function in the same protein kinase cascade (29), which is involved in a variety of pathological conditions, including inflammation, oxidative stress and cell 

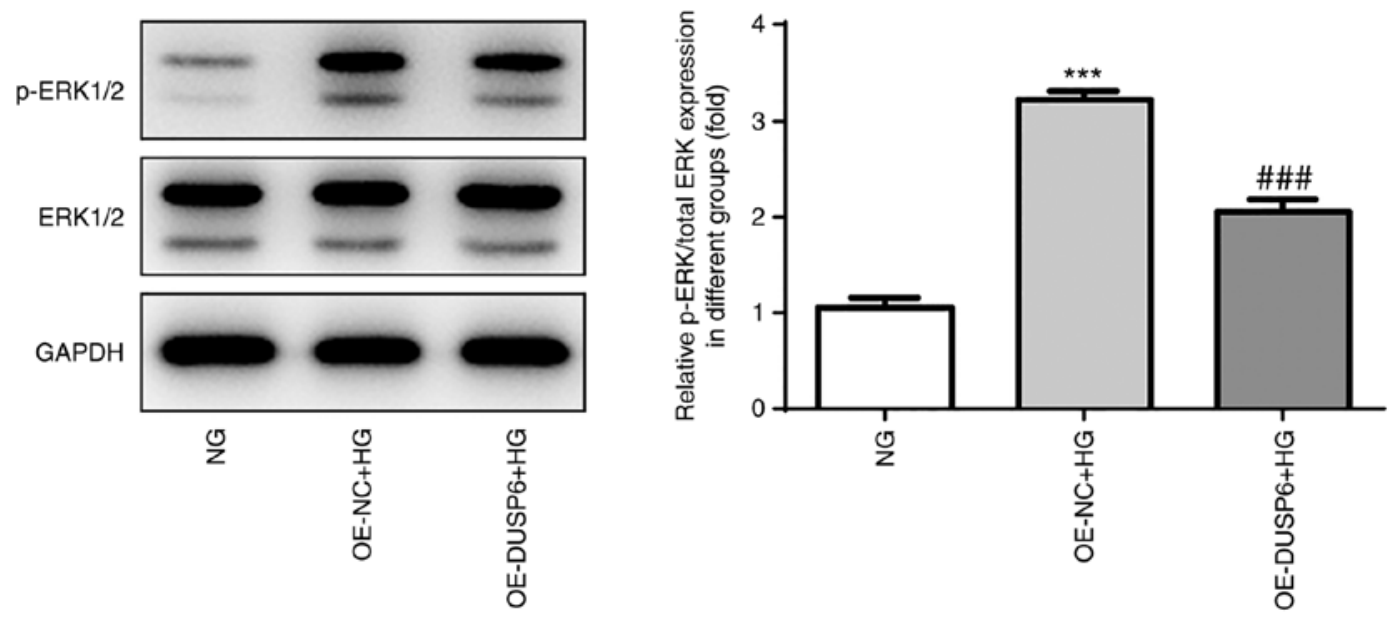

Figure 6. ERK1/2 activation is associated with the role of DUSP6 in murine podocytes. ERK1/2 and p-ERK1/2 expression levels were detected by western blotting. ${ }^{* * *} \mathrm{P}<0.001$ vs. NG; ${ }^{\# \# *} \mathrm{P}<0.001$ vs. OE-NC + HG. DUSP6, dual-specificity phosphatase 6; p, phosphorylated; NG, normal glucose; OE, overexpression; $\mathrm{NC}$, negative control; $\mathrm{HG}$, high glucose.

senescence (30-32). Therefore, the modulatory effect of DUSP6 on ERK1/2 was investigated. Compared with NG conditions, HG conditions increased ERK1/2 phosphorylation, but displayed no effect on the expression levels of total-ERK1/2. Conversely, DUSP6 overexpression suppressed HG-induced ERK1/2 phosphorylation (Fig. 6), suggesting that DUSP6 may prevent HG-induced activation of ERK1/2, which may further affect the role of DUSP6 in MPC5 cells.

\section{Discussion}

Accumulating research has demonstrated that DUSPs serve as tumor suppressors or oncogenes by regulating cell proliferation, migration and apoptosis (12-14), which ultimately influences and determines the fate of specific types of cancer (19). A previous study demonstrated that DUSP1 was tightly associated with a glucose metabolism disorder and glomerular apoptosis via interrupting JNK-mitochondrial fission factor-mitochondrial fission, reducing hyperglycemia-regulated mitochondrial damage and improving renal function (33). Huang et al (34), reported that DUSP26 expression levels in the kidneys of patients with DN were elevated compared with non-diabetic patients. In addition, the progression of DUSP26-regulated DN was largely dependent on the generation of ROS. DUSP9 downregulation in clear cell renal cell carcinoma was also associated with a poor prognosis in a large number of clinical samples (35).

Previous studies demonstrated that DUSP6 was implicated in various types of cancer, including endometrial adenocarcinoma, breast cancer and ovarian cancer (36-39). However, to the best of our knowledge, few studies have investigated the role of DUSP6 in kidney disease, particularly in DN. Therefore, the present study aimed to reveal the regulatory effects of DUSP6 in DN and to determine the pathogenesis of DN to identify effective novel targets for clinical therapy.

Diabetic model mice were established, and it was indicated that DUSP6 expression levels were downregulated in the kidneys of the diabetic model mice, which was accompanied by evident pathological alterations, compared with control mice. Subsequently, in vitro studies using MPC5 cells were conducted to validate the role of DUSP6 following stimulation with D-glucose. MPC5 cells treated with $30 \mathrm{mM}$ D-glucose for $24 \mathrm{~h}$ displayed significantly reduced DUSP6 expression levels compared with control cells. In addition, DUSP6 overexpression inhibited HG-mediated inhibition of cell viability and expression levels of MPC5 cell markers.

A previous study reported that DUSP5 might serve as an endogenous regulator of adipose tissue inflammation (40). Ye et al (41), demonstrated that DUSP9 is a key suppressor of high-fat diet-induced hepatic steatosis and inflammatory responses. Furthermore, DUSP4 ${ }^{-/}$hearts and DUSP4-knockdown cells are more susceptible to oxidant-induced death and tissue injury, indicating a critical defensive role of DUSP4 against oxidative stress (42). In the present study, the results suggested that DUSP6 overexpression attenuated HG-induced oxidative stress and inflammatory responses. In addition, DUSP6 overexpression also inhibited HG-induced podocyte apoptosis.

DUSP6, a negative regulator of ERK1/2, regulates the ERK1/2 signaling cascade (43). The phosphorylation of ERK and p38MAPK is required for human DN (44), contributing to the pathogenesis of DN via stimulation of ROS and inflammatory factors (45). HG rapidly enhances Ras activation, and progressively increases ERK and nuclear c-Jun activation (46), which may be involved in the regulation of inflammation and fibrosis in human renal disease (47). The present study indicated that DUSP6 overexpression decreased ERK1/2 phosphorylation under HG conditions compared with the $\mathrm{HG}+\mathrm{OE}-\mathrm{NC}$ group, which indicated that DUSP6 may exert protective effects against oxidative stress, the inflammatory response and apoptosis in MPC5 cells via altering the activity of ERK1/2. However, the expression levels of c-Jun and p38MAPK were not investigated in the present study; therefore, further investigation into the role of ERK1/2 in MPC5 cell injury using inhibitors to determine the specific mechanisms underlying $\mathrm{DN}$ is required.

In conclusion, the present study demonstrated that DUSP6 expression levels were decreased in the kidneys of DN model mice compared with control mice, which was also indicated by decreased DUSP6 expression levels in HG-induced MPC5 
cells compared with control MPC5 cells. Collectively, the results of the present study suggested that DUSP6 protected MPC5 cells from the inflammatory response and oxidative stress potentially via activation of ERK1/2.

\section{Acknowledgements}

Not applicable.

\section{Funding}

The present study was supported by the College Students' Innovative Entrepreneurial Training Program of Heilongjiang Province (grant no. 20190222020), the Joint Guiding Program of Natural Science Foundation of Heilongjiang Province (grant no. LH2019H060) and the Key Program of Natural Science Foundation of Heilongjiang Province (grant no. ZD2017020).

\section{Availability of data and materials}

The datasets used and/or analyzed during the present study are available from the corresponding author on reasonable request.

\section{Authors' contributions}

LQC and YKW drafted the manuscript and performed the experiments; HYL and GYM analyzed and interpreted the data; and HMZ and GC contributed to the conception and design of the study. All authors read and approved the final manuscript.

\section{Ethics approval and consent to participate}

All experiments were approved by the Biological and Medical Ethics Committee of Jamusi University (approval no. SYXK 2016-014).

\section{Patient consent for publication}

Not applicable.

\section{Competing interests}

The authors declare that they have no competing interests.

\section{References}

1. Farag YM and Al Wakeel JS: Diabetic nephropathy in the Arab Gulf countries. Nephron Clin Pract 119: c317-c322, discussion c322-c323, 2011.

2. Pagtalunan ME, Miller PL, Jumping-Eagle S, Nelson RG, Myers BD, Rennke HG, Coplon NS, Sun L and Meyer TW: Podocyte loss and progressive glomerular injury in type II diabetes. J Clin Invest 99: 342-348, 1997.

3. Susztak K, Raff AC, Schiffer M and Böttinger EP: Glucose-induced reactive oxygen species cause apoptosis of podocytes and podocyte depletion at the onset of diabetic nephropathy. Diabetes 55: 225-233, 2006.

4. Meyer TW, Bennett PH and Nelson RG: Podocyte number predicts long-term urinary albumin excretion in Pima Indians with Type II diabetes and microalbuminuria. Diabetologia 42: 1341-1344, 1999

5. Jefferson JA, Shankland SJ and Pichler RH: Proteinuria in diabetic kidney disease: A mechanistic viewpoint. Kidney Int 74 22-36, 2008.
6. Lu H, Li Y, Zhang T, Liu M, Chi Y, Liu S and Shi Y: Salidroside reduces high-glucose-induced podocyte apoptosis and oxidative stress via upregulating heme oxygenase-1 (HO-1) expression. Med Sci Monit 23: 4067-4076, 2017.

7. Zhu L, Han J, Yuan R, Xue L and Pang W: Berberine ameliorates diabetic nephropathy by inhibiting TLR4/NF- $x$ B pathway. Biol Res 51: 9, 2018.

8. Guo J, Li J, Zhao J, Yang S, Wang L, Cheng G, Liu D, Xiao J, Liu Z and Zhao Z: MiRNA-29c regulates the expression of inflammatory cytokines in diabetic nephropathy by targeting tristetraprolin. Sci Rep 7: 2314, 2017.

9. Haraldsson B: A new era of podocyte-targeted therapy for proteinuric kidney disease. N Engl J Med 369: 2453-2454, 2013.

10. Chen HF, Chuang HC and Tan TH: Regulation of Dual-Specificity Phosphatase (DUSP) Ubiquitination and Protein Stability. Int J Mol Sci 20: 2668, 2019.

11. Farooq A and Zhou MM: Structure and regulation of MAPK phosphatases. Cell Signal 16: 769-779, 2004.

12. Huang CY and Tan TH: DUSPs, to MAP kinases and beyond. Cell Biosci 2: 24, 2012.

13. Lin HP, Ho HM, Chang CW, Yeh SD, Su YW, Tan TH and Lin WJ: DUSP22 suppresses prostate cancer proliferation by targeting the EGFR-AR axis. FASEB J 33: 14653-14667, 2019.

14. Chen YR, Chou HC, Yang CH, Chen HY, Liu YW, Lin TY, Yeh CL, Chao WT, Tsou HH, Chuang HC, et al: Deficiency in VHR/DUSP3, a suppressor of focal adhesion kinase, reveals its role in regulating cell adhesion and migration. Oncogene 36: 6509-6517, 2017.

15. Messina S, Frati L, Leonetti C, Zuchegna C, Di Zazzo E, Calogero A and Porcellini A: Dual-specificity phosphatase DUSP6 has tumor-promoting properties in human glioblastomas. Oncogene 30: 3813-3820, 2011

16. Song H, Wu C, Wei C, Li D, Hua K, Song J, Xu H, Chen L and Fang L: Silencing of DUSP6 gene by RNAi-mediation inhibits proliferation and growth in MDA-MB-231 breast cancer cells: An in vitro study. Int J Clin Exp Med 8: 10481-10490, 2015.

17. Furukawa T, Sunamura M, Motoi F, Matsuno S and Horii A: Potential tumor suppressive pathway involving DUSP6/MKP-3 in pancreatic cancer. Am J Pathol 162: 1807-1815, 2003.

18. Wong VC, Chen H, Ko JM, Chan KW, Chan YP, Law S, Chua D, Kwong DL, Lung HL, Srivastava G, et al: Tumor suppressor dual-specificity phosphatase 6 (DUSP6) impairs cell invasion and epithelial-mesenchymal transition (EMT)-associated phenotype. Int J Cancer 130: 83-95, 2012.

19. Ahmad MK, Abdollah NA, Shafie NH, Yusof NM and Razak SRA: Dual-specificity phosphatase 6 (DUSP6): A review of its molecular characteristics and clinical relevance in cancer. Cancer Biol Med 15: 14-28, 2018.

20. Huang L, Litjens NHR, Kannegieter NM, Klepper M, Baan CC and Betjes MGH: pERK-dependent defective TCR-mediated activation of CD4(+) T cells in end-stage renal disease patients. Immun Ageing 14: 14, 2017.

21. Beaudry K, Langlois MJ, Montagne A, Cagnol S, Carrier JC and Rivard N: Dual-specificity phosphatase 6 deletion protects the colonic epithelium against inflammation and promotes both proliferation and tumorigenesis. J Cell Physiol 234: 6731-6745, 2019.

22. Liao W, Zheng Y, Fang W, Liao S, Xiong Y, Li Y, Xiao S, Zhang $X$ and Liu J: Dual specificity phosphatase 6 protects neural stem cells from $\beta$-amyloid-induced cytotoxicity through ERK1/2 inactivation. Biomolecules 8: 181, 2018.

23. Neumann UH, Ho JSS, Chen S, Tam YYC, Cullis PR and Kieffer TJ: Lipid nanoparticle delivery of glucagon receptor siRNA improves glucose homeostasis in mouse models of diabetes. Mol Metab 6: 1161-1172, 2017.

24. Tang H, Lei CT, Ye C, Gao P, Wan C, Chen S, He FF, Wang YM, $\mathrm{Su} \mathrm{H}$ and Zhang C: MDM2 is implicated in high-glucose-induced podocyte mitotic catastrophe via Notch1 signalling. J Cell Mol Med 21: 3435-3444, 2017.

25. Livak KJ and Schmittgen TD: Analysis of relative gene expression data using real-time quantitative PCR and the 2(-Delta Delta C(T)) Method. Methods 25: 402-408, 2001.

26. Brosius FC III, Alpers CE, Bottinger EP, Breyer MD, Coffman TM, Gurley SB, Harris RC, Kakoki M, Kretzler M, Leiter EH, et al; Animal Models of Diabetic Complications Consortium: Mouse models of diabetic nephropathy. J Am Soc Nephrol 20: 2503-2512, 2009.

27. Hu M, Wang R, Li X, Fan M, Lin J, Zhen J, Chen L and Lv Z: LncRNA MALAT1 is dysregulated in diabetic nephropathy and involved in high glucose-induced podocyte injury via its interplay with $\beta$-catenin. J Cell Mol Med 21: 2732-2747, 2017. 
28. Liu R and Molkentin JD: Regulation of cardiac hypertrophy and remodeling through the dual-specificity MAPK phosphatases (DUSPs). J Mol Cell Cardiol 101: 44-49, 2016.

29. Patterson KI, Brummer T, O'Brien PM and Daly RJ: Dual-specificity phosphatases: Critical regulators with diverse cellular targets. Biochem J 418: 475-489, 2009.

30. Lu N and Malemud CJ: Extracellular signal-regulated kinase: a regulator of cell growth, inflammation, chondrocyte and bone cell receptor-mediated gene expression. Int J Mol Sci 20: 3792, 2019.

31. Karimi P, Gheisari A, Gasparini SJ, Baharvand H, Shekari F, Satarian L and Ader M: Crocetin prevents RPE cells from oxidative stress through protection of cellular metabolic function and activation of ERK1/2. Int J Mol Sci 21: 2949, 2020

32. Zou J, Lei T, Guo P, Yu J, Xu Q, Luo Y, Ke R and Huang D: Mechanisms shaping the role of ERK1/2 in cellular senescence (Review). Mol Med Rep 19: 759-770, 2019.

33. Sheng J, Li H, Dai Q, Lu C, Xu M, Zhang J and Feng J: DUSP1 recuses diabetic nephropathy via repressing JNK-Mff-mitochondrial fission pathways. J Cell Physiol 234: 3043-3057, 2019

34. Huang F, Sheng XX and Zhang HJ: DUSP26 regulates podocyte oxidative stress and fibrosis in a mouse model with diabetic nephropathy through the mediation of ROS. Biochem Biophys Res Commun 515: 410-416, 2019.

35. Wu S, Wang Y, Sun L, Zhang Z, Jiang Z, Qin Z, Han H, Liu Z, Li X, Tang A, et al: Decreased expression of dual-specificity phosphatase 9 is associated with poor prognosis in clear cell renal cell carcinoma. BMC Cancer 11: 413, 2011.

36. Gu W, Yuan Y, Wang L, Yang H, Li S, Tang Z and Li Q Long non-coding RNA TUG1 promotes airway remodelling by suppressing the miR-145-5p/DUSP6 axis in cigarette smoke-induced COPD. J Cell Mol Med 23: 7200-7209, 2019.

37. Fan MJ, Liang SM, He PJ, Zhao XB, Li MJ and Geng F: Dusp6 inhibits epithelial-mesenchymal transition in endometrial adenocarcinoma via ERK signaling pathway. Radiol Oncol 53: 307-315, 2019

38. Li J, Yang C, Yang J and Zou L: Down-regulation of CCL17 in cancer-associated fibroblasts inhibits cell migration and invasion of breast cancer through ERK1/2 pathway. Cancer Manag Res 11: 7439-7453, 2019.

39. James NE, Beffa L, Oliver MT, Borgstadt AD, Emerson JB, Chichester CO, Yano N, Freiman RN, DiSilvestro PA and Ribeiro JR: Inhibition of DUSP6 sensitizes ovarian cancer cells to chemotherapeutic agents via regulation of ERK signaling response genes. Oncotarget 10: 3315-3327, 2019.
40. Habibian JS, Jefic M, Bagchi RA, Lane RH, McKnight RA, McKinsey TA, Morrison RF and Ferguson BS: DUSP5 functions as a feedback regulator of TNF $\alpha$-induced ERK1/2 dephosphorylation and inflammatory gene expression in adipocytes. Sci Rep 7: 12879, 2017.

41. Ye P, Xiang M, Liao H, Liu J, Luo H, Wang Y, Huang L, Chen M and Xia J: Dual-specificity phosphatase 9 protects against nonalcoholic fatty liver disease in mice through ASK1 suppression. Hepatology 69: 76-93, 2019.

42. Barajas-Espinosa A, Basye A, Angelos MG and Chen CA Modulation of p38 kinase by DUSP4 is important in regulating cardiovascular function under oxidative stress. Free Radic Biol Med 89: 170-181, 2015.

43. Vo AH, Swaggart KA, Woo A, Gao QQ, Demonbreun AR, Fallon KS, Quattrocelli M, Hadhazy M, Page PGT, Chen Z, et al: Dusp6 is a genetic modifier of growth through enhanced ERK activity. Hum Mol Genet 28: 279-289, 2019.

44. Sakai N, Wada T, Furuichi K, Iwata Y, Yoshimoto K, Kitagawa K, Kokubo S, Kobayashi M, Hara A, Yamahana J, et al: Involvement of extracellular signal-regulated kinase and p38 in human diabetic nephropathy. Am J Kidney Dis 45: 54-65, 2005.

45. Rane MJ, Song Y, Jin S, Barati MT, Wu R, Kausar H, Tan Y, Wang Y, Zhou G, Klein JB, et al: Interplay between Akt and p38 MAPK pathways in the regulation of renal tubular cell apoptosis associated with diabetic nephropathy. Am J Physiol Renal Physiol 298: F49-F61, 2010.

46. Lin CL, Wang FS, Kuo YR, Huang YT, Huang HC, Sun YC and Kuo YH: Ras modulation of superoxide activates ERK-dependent fibronectin expression in diabetes-induced renal injuries. Kidney Int 69: 1593-1600, 2006.

47. De Borst MH, Prakash J, Melenhorst WB, van den Heuvel MC, Kok RJ, Navis G and van Goor H: Glomerular and tubular induction of the transcription factor c-Jun in human renal disease. J Pathol 213: 219-228, 2007.

(7) $(-)$ This work is licensed under a Creative Commons Attribution-NonCommercial-NoDerivatives 4.0 International (CC BY-NC-ND 4.0) License. 